\title{
A Mixed Scheme for Subgrid-Scale Fluxes in Cloud-Resolving Models
}

\author{
C.-H. Moeng And P. P. Sullivan \\ National Center for Atmospheric Research, * Boulder, Colorado \\ M. F. KHAIROUTDINOV \\ Stony Brook University, Stony Brook, New York \\ D. A. RANDALL \\ Colorado State University, Fort Collins, Colorado
}

(Manuscript received 27 May 2010, in final form 10 August 2010)

\begin{abstract}
A large-domain large-eddy simulation of a tropical deep convection system is used as a benchmark to derive and test a mixed subgrid-scale (SGS) scheme for scalar and momentum fluxes in cloud-resolving models (CRMs). The benchmark simulation resolves a broad range of scales ranging from mesoscale organizations, through gravity waves and individual clouds, down to energy-containing turbulent eddies. A spectral analysis shows that the vertical-velocity kinetic energy peaks at scales from hundreds of meters in the lower cloud layer to several kilometers higher up; these scales are typical grid sizes of today's CRMs. The analysis also shows that a significant portion of the scalar and momentum fluxes in the benchmark simulation are carried by motions smaller than several kilometers (i.e., smaller than a typical grid resolution of CRMs). The broad range of scales of the benchmark simulation is split into two components: filter scale (mimicking CRM resolvable scale) and subfilter scale (mimicking CRM SGS), using filter widths characteristic of a typical CRM grid spacing. The local relationship of the subfilter-scale fluxes to the filter-scale variables is examined. This leads to a mixed SGS scheme to represent the SGS fluxes of scalars and momentum in CRMs. A priori tests show that the mixed SGS scheme yields spatial distributions of subfilter-scale fluxes that correlate much better with those retrieved from the benchmark when compared with an eddy viscosity/diffusivity scheme that is commonly used in today's CRMs.
\end{abstract}

\section{Introduction}

Cloud-resolving models (CRMs) with a grid mesh of a few kilometers are able to resolve deep cloud systems and even large individual clouds, but small clouds and turbulent motions remain subgrid-scale (SGS). SGS physics in CRMs may differ significantly from those in climate models because 1) CRM SGS processes respond locally to resolvable chaotic-like convective motions; 2) convective scales are generally continuous across a wide range of scales with no spectral gap between resolvable

\footnotetext{
* The National Center for Atmospheric Research is sponsored by the National Science Foundation.
}

Corresponding author address: Dr. Chin-Hoh Moeng, MMM Division, NCAR, P.O. Box 3000, Boulder, CO 80307-3000.

E-mail: moeng@ucar.edu and SGS motions; and 3) the motions near the CRM grid cutoff are likely associated with individual clouds and are crucial in transport processes. Therefore, the ensemblemean closure concept used to parameterize SGS processes in conventional climate models is inadequate for representing SGS processes in CRMs. To formulate SGS parameterizations for CRMs requires diagnosing the relationship (or interaction) between small (i.e., CRM SGS) and large (i.e., CRM resolvable) convective eddies. As computer power increases, more numerical weather prediction models will be cloud resolving, and there is a trend toward developing nonhydrostatic global CRMs for regional climate studies. The SGS issue in the context of CRMs needs to be addressed now.

The largest SGS motions in CRMs are those associated with circulations induced by individual clouds or convection; they are critical because scale interaction occurs mostly between neighboring scales. When a model 
grid lies in the energy-containing range, the largest subfilter eddies are found to be important (Leonard 1974; Zhou et al. 2001). Several approaches have been proposed to capture this type of scale interaction-for example, approximate-deconvolution, which is a generalization of nonlinear gradient models (Leonard 1974, 1997; Clark et al. 1979; Stolz et al. 2001), nonlinear eddy viscosity models (Kosovic 1997), the scale-similarity method (Bardina et al. 1980), and partial reconstruction (Chow et al. 2005). A review of these schemes is given by Meneveau and Katz (2000).

In this study we follow the approximate-deconvolution approach first proposed by Leonard (1974) and tested by Clark et al. (1979) to formulate a mixed SGS flux scheme for CRMs. We perform a priori tests of this SGS scheme using a benchmark large-eddy simulation (LES) of a tropical deep convective system that resolves scales from a deep cloud system down to small-scale energy-containing turbulence. The benchmark simulation is described in section 2. In section 3, we use a low-pass filter to split this benchmark flow into two scale ranges: the filter scale (corresponding to CRM resolvable scale) and the subfilter scale (corresponding to CRM SGS). We also retrieve the spatial distributions of the subfilter-scale (SFS) fluxes from the benchmark LES. We then further decompose the SFS fluxes to examine the contribution from the largest SFS motions. This leads to a mixed SGS flux scheme in section 4 . Section 5 presents a priori tests of the mixed scheme against the SFS fluxes of scalars and momentum retrieved from the benchmark simulation. Section 6 summarizes and concludes our study.

\section{Benchmark: A large-eddy simulation of a tropical convective system}

To study the SGS problem in CRMs, scientists (Khairoutdinov et al. 2009) at the National Science Foundation Science and Technology Center for Multiscale Modeling of Atmospheric Processes (CMMAP) performed a large-domain LES of a tropical deep convection system. The numerical domain of $204.8 \mathrm{~km} \times$ $204.8 \mathrm{~km} \times 27 \mathrm{~km}$ is large enough to cover a deep convection system while the grid mesh of $100 \mathrm{~m}$ in horizontal directions with vertically stretched grids varying from 50 to $300 \mathrm{~m}^{1}$ is small enough to resolve energy-containing turbulent eddies. The initial sounding, large-scale forcing, simulation setup, and overall cloud properties were reported in Khairoutdinov et al. (2009). This benchmark simulation was used by Moeng et al. (2009) to examine the PBL structure and to evaluate a commonly used eddy diffusivity model in the lower cloud layer (below $1.2 \mathrm{~km}$ );

\footnotetext{
${ }^{1}$ The $\Delta_{z}$ is smaller than $100 \mathrm{~m}$ below $z \sim 18 \mathrm{~km}$.
}
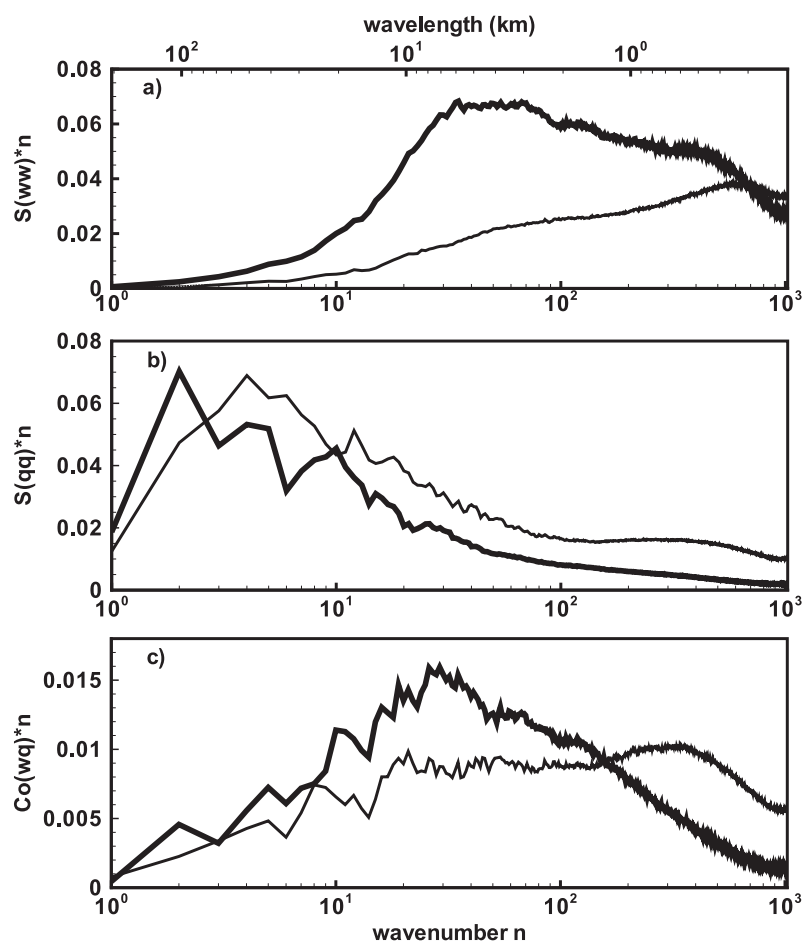

FIG. 1. Two-dimensional $(x, y)$ power spectra of (a) vertical velocity, (b) water mixing ratio, and (c) their cospectra at $z \sim 1 \mathrm{~km}$ (thin curves) and $z \sim 5 \mathrm{~km}$ (thick curves) from the benchmark simulation. The coordinates and their units are chosen such that the areas underneath these curves are equal to the variance and the covariance of the variables in MKS units.

they showed that the spatial correlation coefficient between the SGS vertical flux of moisture estimated by the eddy diffusivity model and that retrieved from the benchmark LES is less than 0.5.

Here we extend the work by Moeng et al. (2009) to study SGS transport of heat, moisture, and momentum over the entire deep convection layer, from the surface to $z=14 \mathrm{~km}$. The SGS includes individual clouds and turbulence (and also small gravity waves and rain-induced vertical motions) that are not resolved by a typical CRM. We derive a mixed SGS scheme to explicitly account for the effect of the largest SGS eddies and carry out a priori tests to gauge its performance.

\section{Spectra and cospectra}

First we perform a two-dimensional (2D in horizontal planes) spectral analysis of the benchmark flow field. The spectra of the vertical velocity $w$ and the water mixing ratio $q$ (water vapor plus nonprecipitating condensed water) and their cospectra at two heights $z \sim 1$ and $5 \mathrm{~km}$, as examples, are shown in Fig. 1. The lower and upper coordinates are the horizontal wavenumber $n=\kappa[L /(2 \pi)]$ and wavelength $\lambda=L / n$, respectively, where $\kappa=\left(k_{x}^{2}+k_{y}^{2}\right)^{1 / 2}, L=204.8 \mathrm{~km}$, and $k_{x}$ and $k_{y}$ are 
wavenumbers in the $x$ and $y$ directions. These spectra are calculated by averaging over wavenumber rings in $k_{x}$ and $k_{y}$ space; they are also averaged over the last $2 \mathrm{~h}$ of the simulation time period, which consists of 24 flow realizations. The coordinates and their units are chosen such that the areas underneath these curves are equal to the variance and the covariance of the variables.

The $w$ variance resides mostly in small scales at $z \sim$ $1 \mathrm{~km}$ (and below, not shown). Higher up, the peak shifts toward larger scales; at $z \sim 5 \mathrm{~km}$, the energy-containing eddies range from several hundred meters up to several kilometers. A typical CRM grid lies in this peak spectral range. The $q$ variance resides mostly at larger scales even in the lower cloud layer.

The $w q$ cospectra (multiplied by $n$ ), however, remain uniform across a broad range of convection scales (several hundred meters up to tens of kilometers), particularly in the lower cloud layer (e.g., $z \sim 1 \mathrm{~km}$ ). This indicates that all convective motions contribute significantly to the vertical moisture flux (and heat flux; not shown). At $z \sim 5 \mathrm{~km}$, the peak contribution to the vertical transport appears at wavelength $\lambda \sim 10 \mathrm{~km}$, which is close to the typical grid resolution of CRMs.

The spectral analysis conveys three important messages: The motions in a deep convection system are continuous in wavenumber space: there is no spectral gap between the "resolvable scale" and "subgrid scale" motions in a convective cloud system, such as those commonly simulated in a CRM. Second, a typical CRM grid of a few kilometers lies in the energy-containing range of the vertical component of kinetic energy, implying strong scale interactions near the CRM grid cutoff that may not be well represented by SGS schemes based on inertial range behaviors. The benchmark also shows that a significant portion of the vertical transport of moisture (and heat; not shown) is carried by motions smaller than several kilometers.

\section{Scale separation of the benchmark LES flow field}

To study the relationship between the CRM resolvable component and the CRM SGS component we partitioned the benchmark LES field into filter-scale (FS; $\tilde{c}$ ) and subfilter-scale $\left(c^{\prime}\right)$ components, where $c$ is a variable of the benchmark simulation. We regard the FS component as a surrogate for the CRM resolvable field and the SFS component as the CRM SGS field. ${ }^{2}$

\footnotetext{
${ }^{2}$ In strict terms, SGS is not the same as SFS, particularly when a smooth filter, Gaussian or top hat, is used to define the scale separation. As shown later, SFS consists of motions that are actually larger than the filter width or the grid resolution. Nevertheless, we use the terms SFS and SGS interchangeably. For a discussion of the differences between SFS and SGS we refer readers to Zhou et al. (2001) or Gullbrand and Chow (2003).
}

\section{a. Low-pass-filtering process}

We perform the scale separation using a low-pass filter. The FS $(\tilde{c})$ and SFS $\left(c^{\prime}\right)$ fields at each grid point are defined as

$$
\begin{aligned}
\tilde{c}(x, y) & =\iint c G(x-\eta, y-\zeta) d \eta d \zeta \quad \text { and } \\
c^{\prime}(x, y) & =c(x, y)-\tilde{c}(x, y) .
\end{aligned}
$$

Here we use a Gaussian filter function,

$$
G(x-\eta, y-\zeta)=\frac{6}{\pi} \frac{1}{\Delta_{f}^{2}} \exp \left\{-6\left[(x-\eta)^{2}+(y-\zeta)^{2}\right] / \Delta_{f}^{2}\right\}
$$

or, in terms of wavenumber,

$$
G(\kappa)=\exp \left[-\left(\kappa^{2} \Delta_{f}^{2} / 24\right)\right]
$$

where $\kappa$ is the 2D horizontal wavenumber and $\Delta_{f}$ is the filter width in both the $x$ and $y$ directions. The low-pass filtering is performed using a 2D FFT in each horizontal plane. As shown by Tong et al. (1998), 2D filtering in $x$ and $y$ is a good approximation of 3D volume (or grid volume) averaging for atmospheric turbulence in the PBL.

We chose the Gaussian filter for two reasons. First, it behaves similarly to a top-hat filter (or area averaging) (Leonard 1974; Horst et al. 2004), and the latter is implicitly assumed in finite-differencing CRMs. Second, unlike the sharp cutoff filter, this smooth filter does not generate numerical oscillations near the cutoff scale in physical space. For a practical check, we also performed area averaging (i.e., using a running mean) in defining FS variables in Eq. (1); as expected the results are very similar to those with a Gaussian filter. For example, averaging over each $4 \mathrm{~km} \times 4 \mathrm{~km} x-y$ subdomain produces results very similar to those of Gaussian filtering with $\Delta_{f}=4 \mathrm{~km}$.

We regard the filter width $\Delta_{f}$ as the CRM's grid "resolution" and note that the effective resolution is often 4-6 times the actual grid spacing because of numerical errors (Pielke 2001). Hence, our FS and SFS solutions with $\Delta_{f}=4 \mathrm{~km}$ may correspond to resolvable and SGS fields in a CRM with a horizontal grid spacing of $\sim 1 \mathrm{~km}$. Other than this concept, we do not account for the effects of numerical errors in developing the SGS scheme, because these effects are beyond the scope of this paper.

It is important to note that a filter width of $4 \mathrm{~km}$ (or $10 \mathrm{~km}$ as shown later) examined in this study is much larger than the horizontal grid mesh $(100 \mathrm{~m})$ of the benchmark simulation; it is also much smaller than the total domain size. Thus, the retrieved SFS feature and 

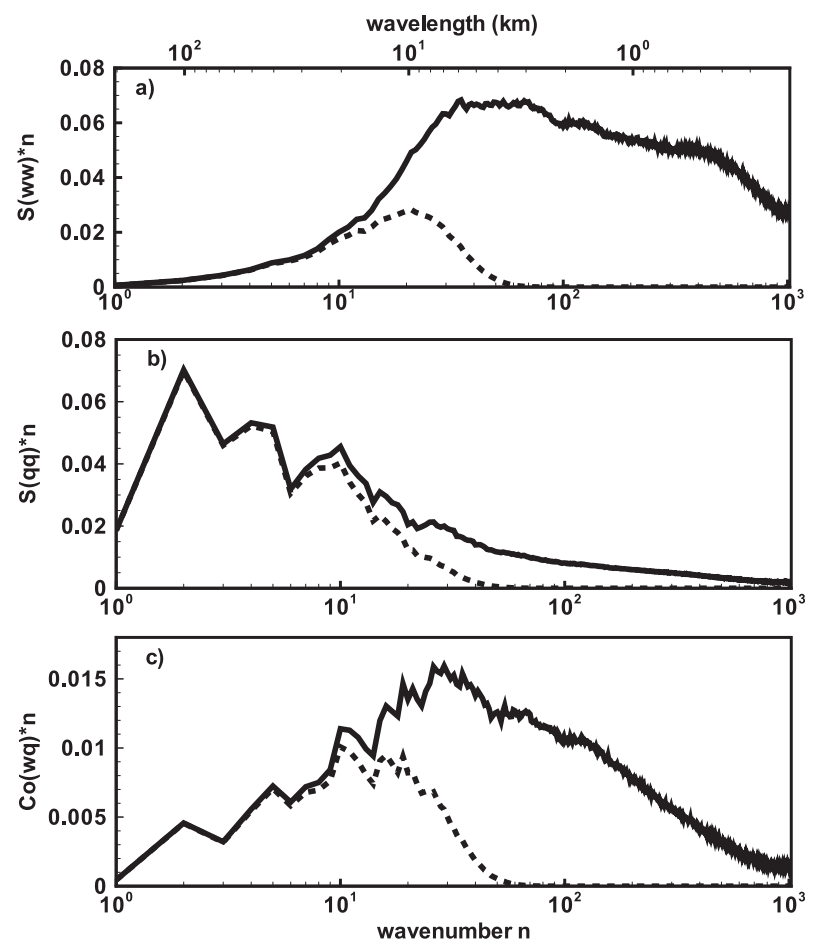

FIG. 2. Two-dimensional power spectra of (a) vertical velocity, (b) water mixing ratio, and (c) their cospectra at $z \sim 5 \mathrm{~km}$, before (solid lines) and after (dashed lines) low-pass filtering of the benchmark simulation, with $\Delta_{f}=4 \mathrm{~km}$.

its relationship to the FS are not strongly affected by the uncertainties due to numerics and SGS of the benchmark simulation.

Figure 2 shows the 2D spectra and cospectrum of the $w$ and $q$ fields before and after low-pass filtering at $z \sim$ $5 \mathrm{~km}$ with $\Delta_{f}=4 \mathrm{~km}$. The area below the dotted curves belongs to FS, while the area between the two curves is the SFS contribution. With $\Delta_{f}=4 \mathrm{~km}$, about $80 \%$ of the $w$ variance resides in the SFS; only a small fraction is retained in the FS. While most of the $q$ variance remains in the FS, a large portion of the covariance between $w$ and $q$ is SFS. For example, at $z \sim 5 \mathrm{~km}$, more than half of the vertical flux of moisture is carried by SFS motions.

Note that with a smooth filter a large amount of SFS variance and flux actually resides at scales larger than the filter width $\Delta_{f}$ (i.e., the areas between the two curves with wavelength $\lambda>4 \mathrm{~km}$ in Fig. 2). We will come back to this point later.

\section{b. Vertical profiles of fluxes before and after low-pass filtering}

Figure 3 shows vertical profiles of horizontally averaged fluxes before and after low-pass filtering with $\Delta_{f}=$ 4 and $10 \mathrm{~km}$. (Hereinafter, the analysis is performed on data volumes archived near the end of the benchmark simulation.) The unfiltered fluxes are calculated from the benchmark flow excluding the subgrid fluxes below the LES grid. As anticipated, the magnitudes of the horizontally averaged fluxes are systematically reduced by filtering. The dotted and dash-dotted curves correspond to fluxes typically resolved by a CRM with a grid resolution of 4 and $10 \mathrm{~km}$, respectively. The differences between the total and the FS fluxes are the SFS fluxes, and they are the main focus of this study.

However, the horizontally averaged SFS fluxes are not what is needed in a CRM. It is the local SFS fluxes that appear in the governing equations of a CRM. Hence, we next look into the local distributions of the SFS fluxes.

\section{c. Spatial distributions of the SFS fluxes}

We retrieve the spatial distributions of the SFS fluxes (using the vertical flux of a scalar $c$ as example) from the benchmark LES using the following definition:

$$
\tau_{w c}=\widetilde{w c}-\tilde{w} \tilde{c}
$$

which appears in the conservation equation for $\tilde{c}$.

The above expression can be further decomposed into three terms, each in square brackets:

$$
\begin{aligned}
\tau_{w c}= & {[\widetilde{\tilde{w} \tilde{c}}-\tilde{\tilde{w}} \tilde{\tilde{c}}]+\left[\widetilde{\tilde{w} c^{\prime}}+\widetilde{w^{\prime} \tilde{c}}-\widetilde{\tilde{w}} \widetilde{c^{\prime}}-\widetilde{w^{\prime}} \tilde{\bar{c}}\right] } \\
& +\left[\widetilde{w^{\prime} c^{\prime}}-\widetilde{w^{\prime}} \widetilde{c^{\prime}}\right],
\end{aligned}
$$

following Germano (1986), who modified the original Leonard decomposition (Leonard 1974) to satisfy Galilean invariance and filter-independent properties. The first term in Eq. (6) is the modified-Leonard term, or just the Leonard term (L). This term depends only on filtered scales (i.e., the tilde variables). It is also referred to as the resolved subfilter scale by Zhou et al. (2001) and Chow et al. (2005) because it accounts for the part of SFS that is actually larger than the filter width as a result of smooth filtering (e.g., the SFS contribution between the two spectral curves with wavelength $\lambda>4 \mathrm{~km}$ in Fig. 2). The second term is a modified-cross term, or just cross term $(\mathrm{C})$, which represents the interaction between the FS and SFS. The last term is the Reynolds (R) term, which depends only on the prime variables. In this paper, we loosely refer to both the $\mathrm{L}$ and $\mathrm{C}$ terms as being representative of contributions from the "largest SFS (or SGS) eddies" (i.e., the SFS eddies that have scales similar to the horizontal resolution of a CRM). As shown in Fig. 2, these largest SFS eddies reside in the range of $w$-energy-containing scales and they contribute significantly to the vertical moisture flux (and other fluxes; not shown here). 

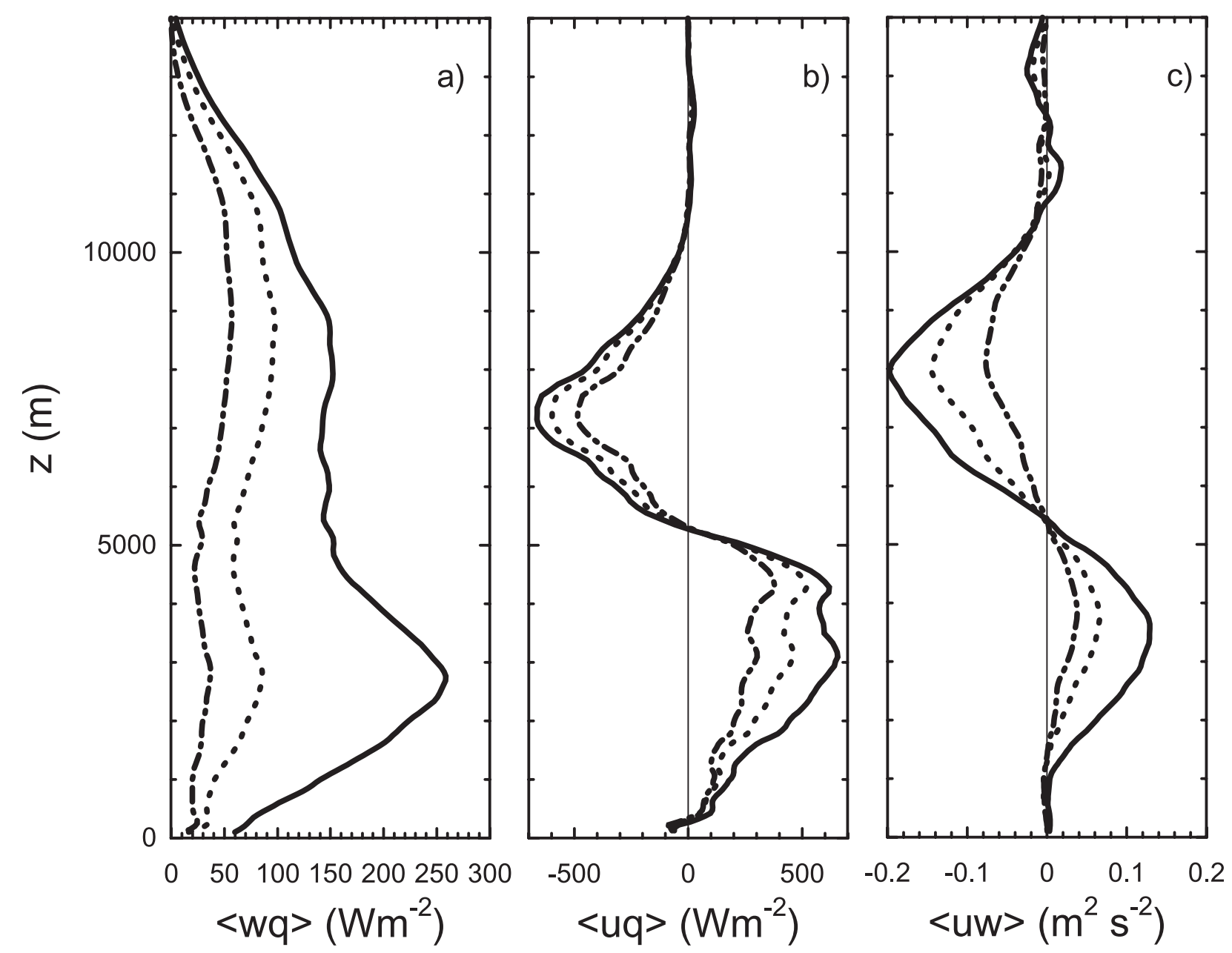

FIG. 3. Vertical profiles of the horizontally averaged (a) vertical fluxes of moisture, (b) horizontal fluxes of moisture, and (c) vertical fluxes of zonal wind. The solid curves are from the unfiltered LES field, the dotted curves are from low-pass-filtered LES with $\Delta_{f}=$ $4 \mathrm{~km}$, and the dash-dotted curves are from low-pass-filtered LES with $\Delta_{f}=10 \mathrm{~km}$. Angle brackets represent averages over the entire horizontal plane.

Sullivan et al. (2003) studied the contributions of the L and $\mathrm{C}$ terms to SFS stress tensors using a set of turbulence data collected from the Horizontal Array Turbulence Study field program in the atmospheric surface layer. They show that when $\Lambda_{w} / \Delta_{f} \sim 1$, where $\Lambda_{w}$ is the $w$-energy peak scale, the sum of the $\mathrm{L}$ and $\mathrm{C}$ terms can contribute as much as one-fifth of the total SFS momentum flux. When $\Lambda_{w} / \Delta_{f} \sim 2$, their contributions increase to about one-half of the total SFS flux. The $w$-energy peak in the simulated deep convection system covers a broad range of scales (Fig. 1a), and hence it is not easy to identify a characteristic length scale of $\Lambda_{w}$. Nonetheless, the scale where the $w$ energy peaks is on the order of several kilometers. This is one of the reasons why we chose filter widths of $\Delta_{f}=4$ and $10 \mathrm{~km}$ for analysis. As mentioned before, we also chose this range of filter widths because they are close to the effective resolution of a typical CRM and because the motions near these filter widths are not strongly affected by the uncertainties due to SGS and numerics of our benchmark simulation.

Using the definitions of the L, C, and R terms in Eq. (6), we retrieved each term from the benchmark LES field. Figure 4 displays an example of the horizontal distributions of the total SFS moisture flux and each of its component terms, at $z \sim 5 \mathrm{~km}$ with $\Delta_{f}=4 \mathrm{~km}$. The contours in Fig. 4b (L term), Fig. 4c (C term), and Fig. 4d ( $\mathrm{R}$ term) sum to the total shown in Fig. 4a. To show the detailed structures of each term, we use different color scales: $[-300,22000] \mathrm{W} \mathrm{m}^{-2}$ for Fig. $4 \mathrm{a},[-100,4000]$ $\mathrm{W} \mathrm{m}{ }^{-2}$ for Fig. 4b, $[-1000,6000] \mathrm{W} \mathrm{m}^{-2}$ for Fig. 4c, and $[-200,16000] \mathrm{W} \mathrm{m}^{-2}$ for Fig. 4d. First, we notice that the SFS moisture fluxes fluctuate greatly in space. The large variation is associated with the circulations of the 

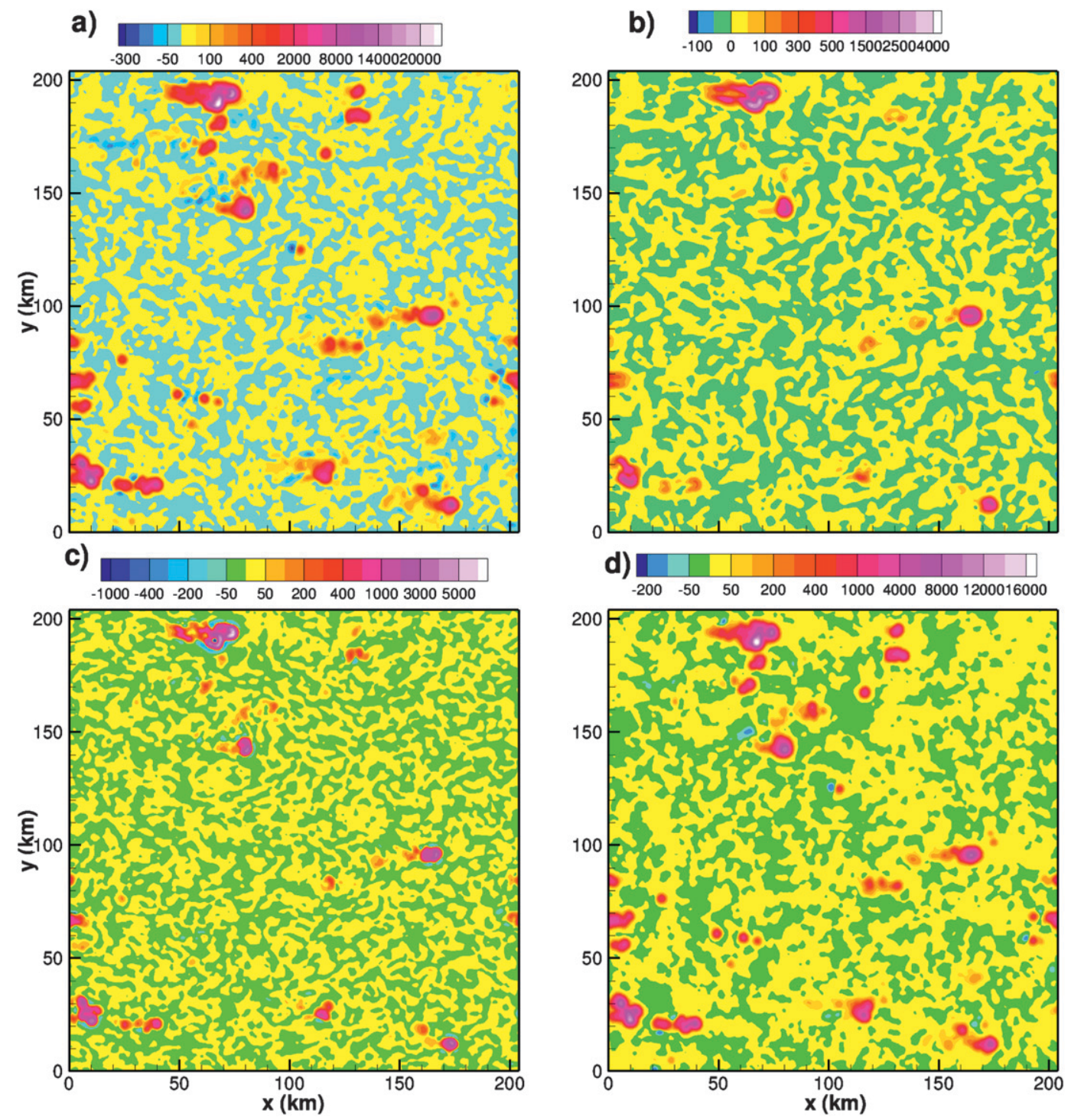

FIG. 4. Horizontal distributions of the SFS vertical moisture fluxes $\left(\mathrm{W} \mathrm{m}^{-2}\right)$ at $z \sim 5 \mathrm{~km}$ retrieved from the LES: (a) total SFS flux, (b) L term, (c) C term, and (d) R term. Warm colors represent positive values, and cold colors show negative values.

convective system. As shown in Moeng et al. (2009), these large fluctuations also exist in the PBL and are associated with precipitation-induced cold pools.

All three terms have spatial distributions that are similar to the total SFS flux, albeit with different magnitudes. The $\mathrm{R}$ term dominates the total SFS flux; its largest local flux is about $16000 \mathrm{~W} \mathrm{~m}^{-2}$. However, the $\mathrm{L}$ and $\mathrm{C}$ terms also contribute a significant fraction to the total SFS flux.

To show the contributions of the individual terms at all heights, we compute their horizontal averages and compare them with the total in Fig. 5. (The SFS fluxes are nearly zero above $z \sim 14 \mathrm{~km}$, which is above the cloud-top height, and hence are not shown.) The R term contributes about $70 \%$ of the horizontally averaged SFS flux, while the sum of the $\mathrm{L}$ and $\mathrm{C}$ terms contributes about $30 \%$, for the case with $\Delta_{f}=4 \mathrm{~km}$.

We next examine the spatial correlations of the $\mathrm{L}, \mathrm{C}$, and R terms with the total SFS flux in Fig. 6a. This is done by correlating the fields in Figs. 4b-d, respectively, point by point with that in Fig. 4a, normalizing by their standard deviations, and averaging them over horizontal planes. All terms in Eq. (6) correlate well with the total SFS flux, with the coefficient being larger than 0.8 in most of the deep cloud layer. Figure $6 \mathrm{~b}$ shows the correlation coefficient between the $\mathrm{L}$ term and the $\mathrm{C}$ term, 


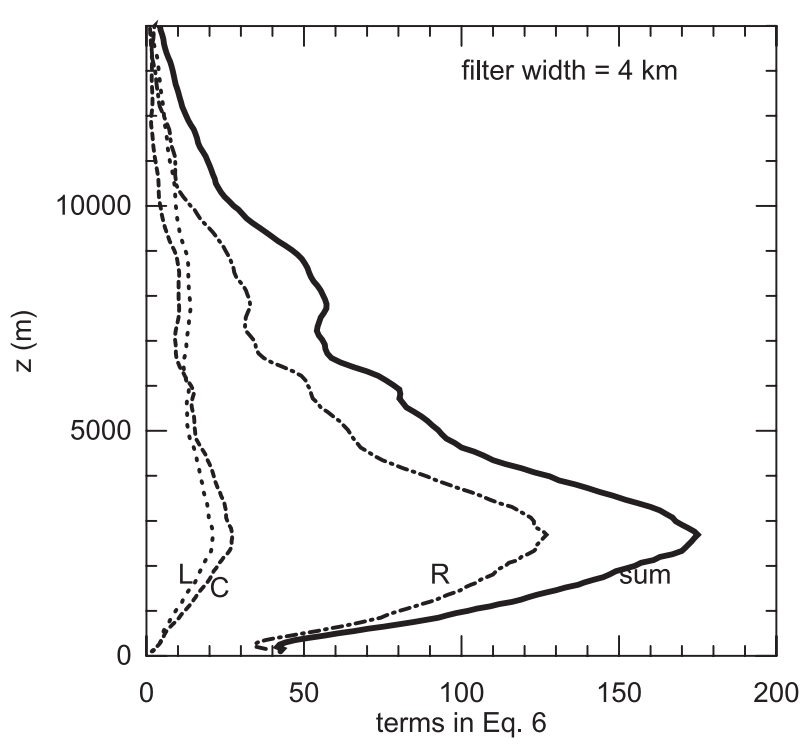

FIG. 5. Vertical distributions of the horizontally averaged fluxes ( $\mathrm{W} \mathrm{m}^{-2}$ ) of each term on the right-hand side of Eq. (6) in comparison with their sum calculated from the low-pass-filtered LES with $\Delta_{f}=4 \mathrm{~km}$.

as well as the ratio of their standard deviations, $\mathrm{sd}(\mathrm{L}) /$ $\operatorname{sd}(\mathrm{C})$. The correlation coefficient between these two terms is around 0.8 , and the ratio of their fluctuation levels is between 0.6 and 1.5. An examination of the case with $\Delta_{f}=10 \mathrm{~km}$ shows similar results. Thus, we will assume $\mathrm{C} \sim \mathrm{L}$ in deriving the mixed SGS scheme in section 4.

The main reason for examining the individual contributions and spatial correlations of each term in Eq. (6), separately, is the following: The $\mathrm{R}$ and $\mathrm{C}$ terms involve the SFS (the prime variables) and require closure assumptions in order to model them, whereas the $\mathrm{L}$ term involves only the tilde variables (corresponding to resolvable variables in CRMs). Because the L term contains only FS variables, which are smooth on the grid scale, it can be adequately approximated using a Taylor series expansion without making any closure assumption, which is illustrated next.

\section{d. The effect of the largest SFS eddies}

Numerous engineering studies have recovered the $\mathrm{L}$ term in Eq. (6) using different approaches. We note that it is equivalent to the scale similarity term first proposed by Bardina et al. (1980) and is also closely linked to deconvolution and filter inversion models (Chow et al. 2005; Stolz et al. 2001); it also appears as the first term in the tensor diffusivity model developed by Leonard (1997). The tensor diffusivity model inverts the filtering by using a Taylor series expansion of the velocity and scalar fields, which is the approach we follow.
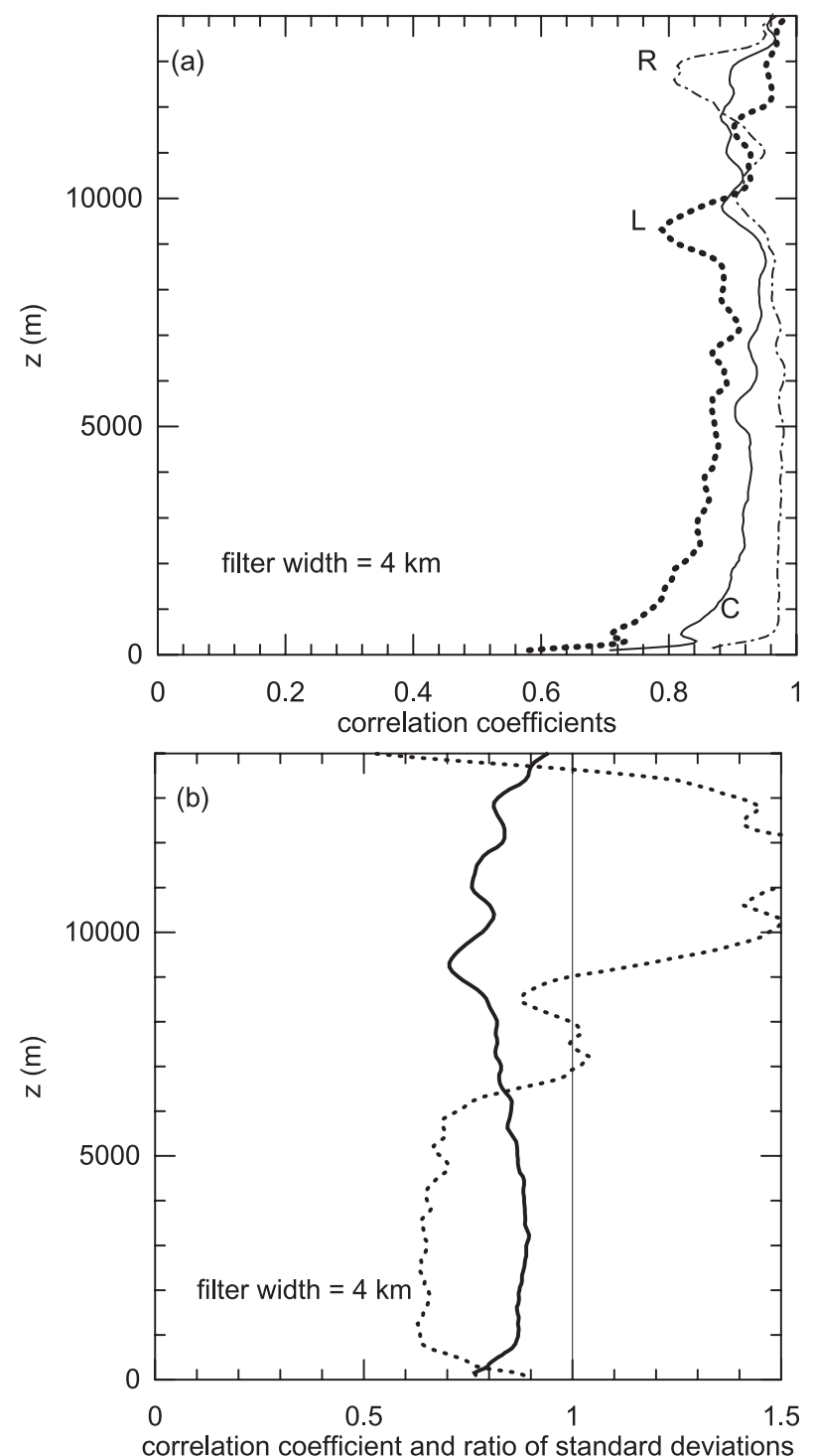

FIG. 6. (a) Correlation coefficients of each term of the right-hand side of Eq. (6) with the total SFS flux $\tau_{w q}$, and (b) correlation coefficient (solid curve) between the $\mathrm{L}$ term and the $\mathrm{C}$ term and the ratio of their standard deviations (dotted curve) retrieved from the LES with $\Delta_{f}=4 \mathrm{~km}$.

Applying a Taylor series expansion to the double-tilde term $\tilde{\tilde{w}}$ (see the appendix), we have

$$
\tilde{\tilde{w}} \approx \tilde{w}+\frac{\Delta_{f}^{2}}{24}\left(\frac{\partial^{2} \tilde{w}}{\partial x \partial x}+\frac{\partial^{2} \tilde{w}}{\partial y \partial y}\right)+\text { higher-order terms. }
$$

A similar expansion for other double-tilde terms, $\tilde{\tilde{c}}$ and $\widetilde{\tilde{w} \tilde{c}}$, can be introduced. Only $x$ and $y$ derivatives appear in Eq. (7) because the filtering is performed in horizontal planes. By using Eq. (7), rearranging the terms, 


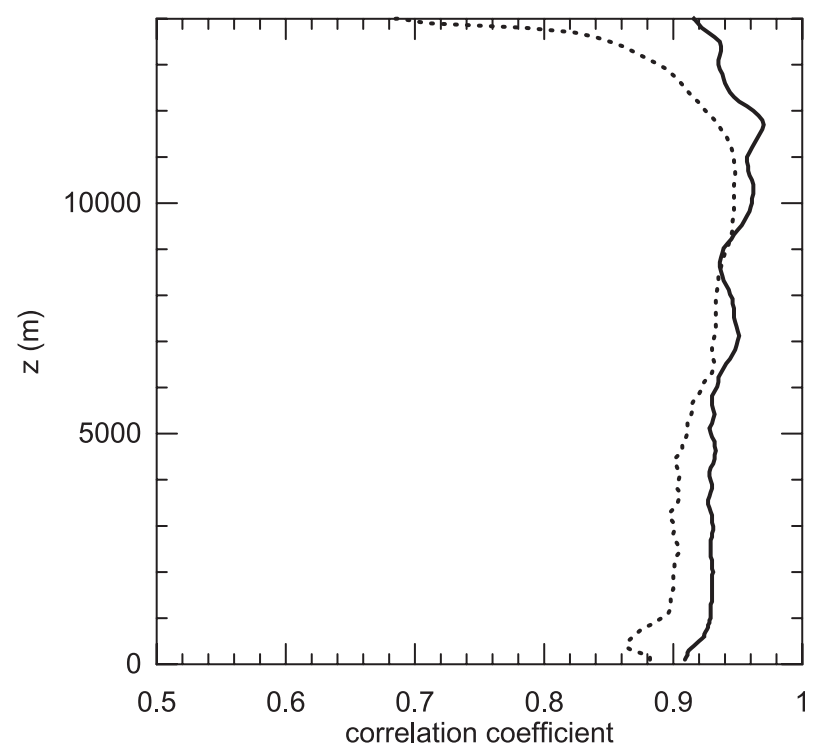

FIG. 7. Correlation coefficients between the right-hand and lefthand sides of Eq. (8), retrieved from the LES field using $\Delta_{f}=4$ (solid curves) and 10 (dotted curves) $\mathrm{km}$.

and neglecting the higher-order terms, the Leonard term can be approximated as

$$
\widetilde{\tilde{w} \tilde{c}}-\tilde{\tilde{w}} \tilde{\tilde{c}} \approx\left(\frac{\Delta_{f}^{2}}{12}\right)\left(\frac{\partial \tilde{w}}{\partial x} \frac{\partial \tilde{c}}{\partial x}+\frac{\partial \tilde{w}}{\partial y} \frac{\partial \tilde{c}}{\partial y}\right) .
$$

The right-hand side of the above expression has a clear computational advantage over its left-hand side in that it can be computed without any additional filtering. All CRMs readily have available derivatives of the resolvable variables, which appear in Eq. (8), and hence it is inexpensive to include Eq. (8) in an SGS scheme.

However, we need to verify the adequacy of this oneterm Taylor series approximation for a deep convection system: We calculate both sides of Eq. (8) from the LES for $c=q$, correlate them point by point in each horizontal plane, and show their correlation coefficients in Fig. 7 . The coefficients are nearly 0.9 throughout most of the deep convection layer for the two filter widths examined here. (The small correlation coefficient at $z \sim 14 \mathrm{~km}$, which is above the cloud-top height, for $\Delta_{f}=$ $10 \mathrm{~km}$ is due to the negligibly small SFS flux and hence is not of concern.)

\section{A mixed SGS transport scheme}

A commonly used SGS transport scheme in CRMs (e.g., Klemp and Wilhelmson 1978; Khairoutdinov and Randall 2003) is the Smagorinsky-Deardorff type- $K$ model wherein a scalar SGS flux is expressed as

$$
\tau_{w c}=-K_{h} \frac{\partial \tilde{c}}{\partial z}
$$

and, following Deardorff (1980), the eddy diffusivity $K_{h}$ is related to the eddy viscosity $K_{m}$ as

$$
K_{h}=[1+(2 l / \Delta)] K_{m},
$$

where

$$
K_{m}=c_{K} \ell \sqrt{e}
$$

Here $e$ is the SGS turbulent kinetic energy (TKE), $\ell$ is an SGS length scale, $c_{K}$ is a diffusion coefficient, and $\Delta$ is the grid resolution. In the following a priori tests, the SGS TKE $e$ was taken from the retrieved SFS TKE, and $\Delta$ was set equal to $\left(\Delta_{f}^{2} \Delta_{z}\right)^{1 / 3}$, where $\Delta_{z}$ is the vertical grid size of the benchmark simulation. The coefficient $c_{K}$ was tuned to 0.4 so that the modeled SGS moisture flux $\tau_{w q}$ has a horizontally averaged profile that is comparable to the retrieved one. The constant was then kept the same for later a priori tests of other fluxes.

The SGS length scale $\ell$ is the minimum of two length scales

$$
\ell=\min \left[0.76 e^{1 / 2}\left(\frac{g}{\theta_{0}} \frac{\partial \tilde{\theta}_{l}}{\partial z}\right)^{-1 / 2} ; \Delta\right],
$$

if $\partial \tilde{\theta}_{l} / \partial z$ is positive, where $\tilde{\theta}_{l}$ is the resolvable liquidwater potential temperature (Deardorff 1980). We also applied a wall damping correction; that is, $\ell$ was limited to $k z / c_{S}$ near the surface (Sullivan et al. 1994), where $k=$ 0.4 is the von Kármán constant and $c_{S}=0.18$ is the Smagorinsky constant. The stability and wall corrections to the SGS length scale enhanced the $K$-model performance in the PBL, as shown later.

Our main modification to the $K$ model in this study is to include the effect of the largest SFS eddies, namely the $\mathrm{L}$ and $\mathrm{C}$ terms, via the expression shown in Eq. (8). Although this expression was derived based on the $\mathrm{L}$ term, we apply it to the $\mathrm{C}$ term as well because Figs. $4 \mathrm{~b}$, $4 \mathrm{c}, 5$, and 6 suggest that the two terms behave similarly.

A mixed SGS model for a scalar flux is then

$$
\tau_{w c}=-K_{h} \frac{\partial \tilde{c}}{\partial z}+2\left(\frac{\Delta_{f}^{2}}{12}\right)\left(\frac{\partial \tilde{w}}{\partial x} \frac{\partial \tilde{c}}{\partial x}+\frac{\partial \tilde{w}}{\partial y} \frac{\partial \tilde{c}}{\partial y}\right),
$$

where the second term is referred to as the $\mathrm{L}+\mathrm{C}$ term (hence the factor 2) and the first term is the commonly used $\mathrm{K}$ term. This mixed modeling approach is not new; it has been used in the engineering community (e.g., Bardina et al. 1980; Piomelli et al. 1988; Zang et al. 1993) 

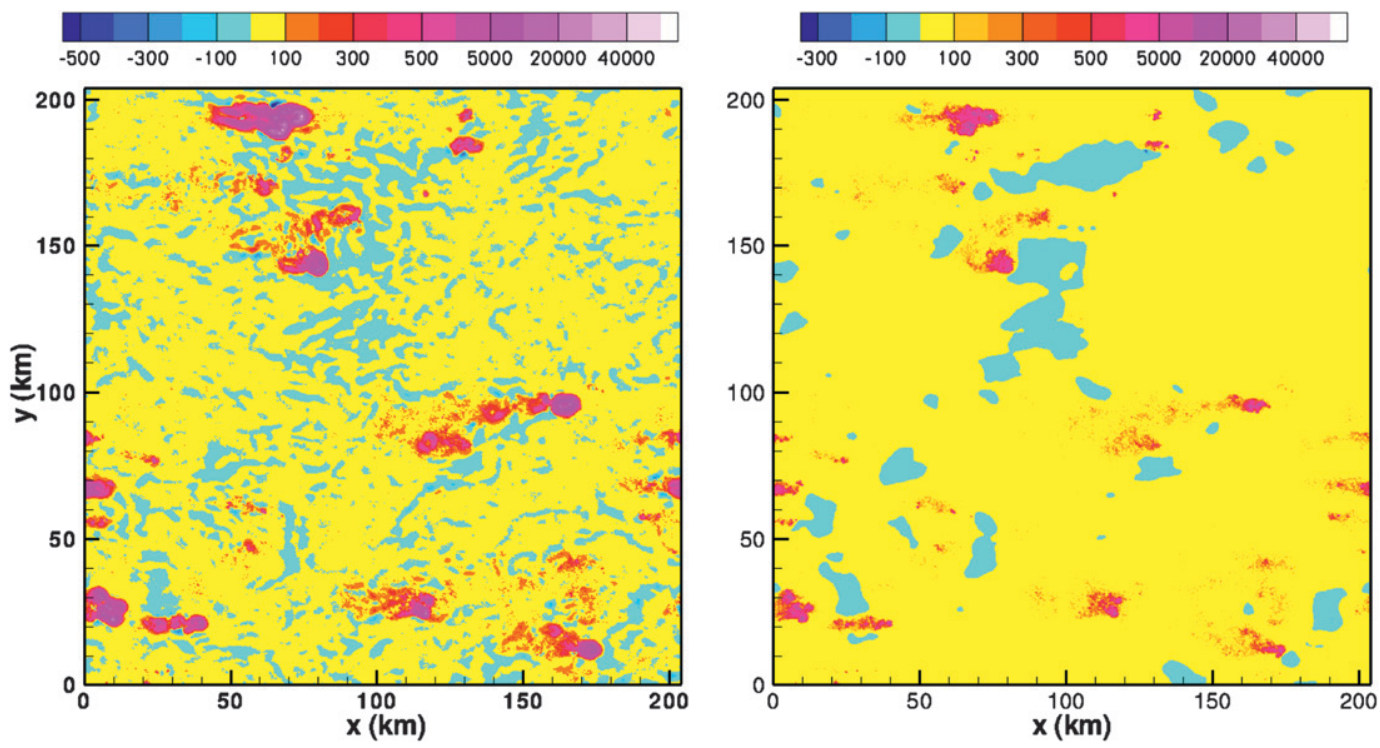

FIG. 8. Horizontal distributions of the modeled SFS vertical moisture fluxes $\left(\mathrm{W} \mathrm{m}^{-2}\right)$ at $z \sim 5 \mathrm{~km}$ (left) from the mixed scheme and (right) from just the $\mathrm{K}$ term in Eq. (13).

for modeling the SFS stresses in LES. It has also been applied to LES of the PBL (Kosovic 1997; Chow et al. 2005). Those were applications to turbulence simulations. Here we apply it to simulations of deep convection systems, which include nonturbulent motions such as mesoscale cloud organizations and gravity waves as well as turbulence.

Some studies (e.g., Carati et al. 2001) suggest that the $\mathrm{K}$ term may be proper to model the $\mathrm{R}$ term in Eq. (6) because the $R$ term is representative of the effect of smaller SFS eddies. But we show in Figs. 4-6 that the R term actually behaves similar to the $\mathrm{L}$ and $\mathrm{C}$ terms and it dominates the total SFS flux. We will also show later that the $K$ model correlates poorly with the R term. Nevertheless, the K term in Eq. (13) is kept not only because it is widely used in CRMs but also because it can properly dissipate turbulent kinetic energy that is required for numerical stability in models (Bardina et al. 1980; Liu et al. 1999; Sullivan et al. 2003).

\section{A priori tests of the mixed subgrid-scale scheme}

We next perform a priori tests on the mixed SGS scheme in representing SGS fluxes using filter widths characteristic of a typical CRM grid spacing.

\section{a. Vertical SGS fluxes of scalars}

The modeled $\tau_{w q}$ with and without the second term in Eq. (13) at $z \sim 5 \mathrm{~km}$ with $\Delta_{f}=4 \mathrm{~km}$ are shown in Fig. 8 and compared with the SFS flux derived from the LES, which is Fig. 4a. The comparison shows that the $K$ model alone (right panel) underpredicts the spatial variations and the area covered by negative fluxes. With the additional $\mathrm{L}+\mathrm{C}$ term, the modeled SFS fluxes are much improved: Its spatial distribution is much closer to the retrieved-flux distribution.

For statistical measures, we compute the spatial (point by point) correlations between the modeled and the LES-retrieved SFS fluxes for two filter widths, shown in Fig. 9. The addition of the $\mathrm{L}+\mathrm{C}$ term increases the

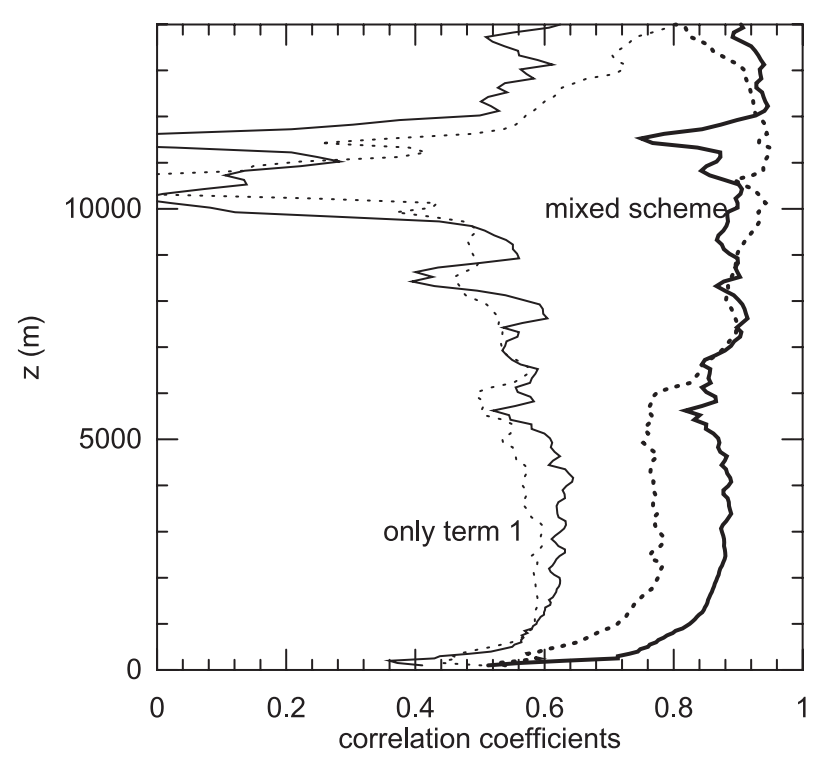

FIG. 9. Correlation coefficients between the modeled and the LESretrieved $\tau_{w q}$ with $\Delta_{f}=4$ (solid curves) and 10 (dotted curves) $\mathrm{km}$. 


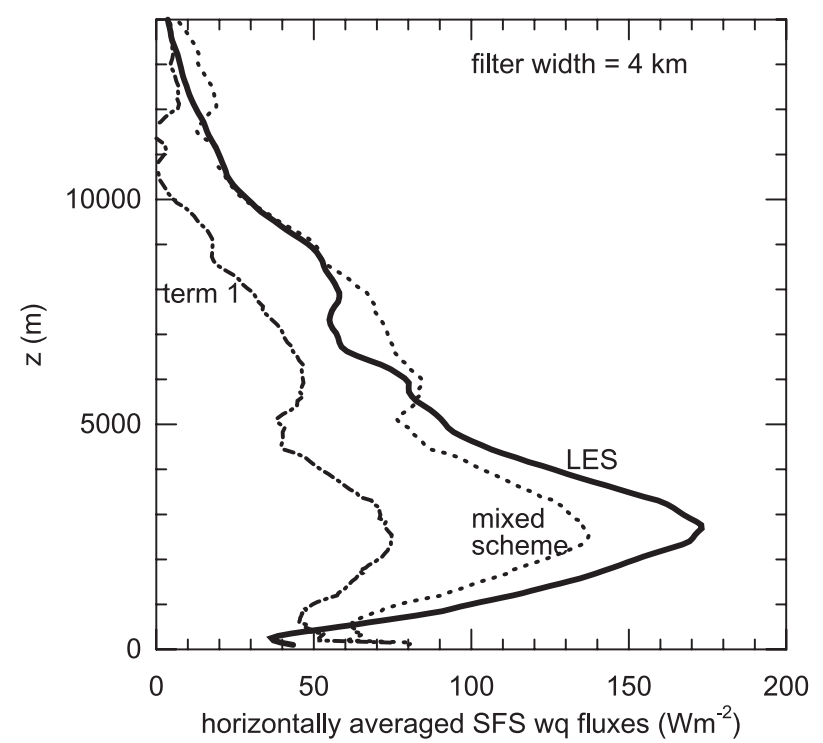

FIG. 10. Vertical distributions of the horizontally averaged $\tau_{w q}$ from Eq. (13) in comparison with the LES-retrieved $\left\langle\tau_{w q}\right\rangle$ with $\Delta_{f}=4 \mathrm{~km}$.

correlation coefficients from about 0.6 to about 0.9 over the entire deep-cloud layer except in the PBL. In the PBL, the mixed SGS scheme (for the filter widths examined here) does not perform as well. However, relative to the $K$ model with a constant $\ell$ tested by Moeng et al. (2009), the $K$ model with the stability correction [Eq. (12)] increases the correlation coefficients from less than 0.2 (see Fig. 12 in Moeng et al. 2009) to about 0.5 at $z \sim 400 \mathrm{~m}$. The addition of the $\mathrm{L}+\mathrm{C}$ term further increases the correlation coefficients to $\sim 0.75$ at that height for the $\Delta_{f}=4 \mathrm{~km}$ case.

The horizontally averaged $\left\langle\tau_{w q}\right\rangle$ predicted by just the $\mathrm{K}$ term and by the mixed scheme are compared in Fig. 10 for $\Delta_{f}=4 \mathrm{~km}$. The flux profile from the mixed scheme compares well to the LES-retrieved profile, partly because the diffusion coefficient $c_{K}$ in Eq. (11) is tuned to 0.4. However, it is important to note that this constant will be used for all other tests without further tuning.

We also examined the vertical flux of liquid potential temperature (i.e., heat flux); the result is similar to the moisture flux and hence is not shown here.

\section{b. Horizontal SGS fluxes of scalars}

For SFS horizontal fluxes, $\tau_{u q}$, we also examine the $\mathrm{L}$, $\mathrm{C}$, and $\mathrm{R}$ terms in Eq. (6) retrieved from the low-passfiltered LES field. Figure 11 shows that these three terms contribute almost equally to the horizontally averaged $\left\langle\tau_{u q}\right\rangle$ for $\Delta_{f}=4 \mathrm{~km}$. Again, the $\mathrm{L}$ and $\mathrm{C}$ terms behave similarly.

The mixed SGS scheme for the horizontal flux ( $x$ direction as an example) of moisture is

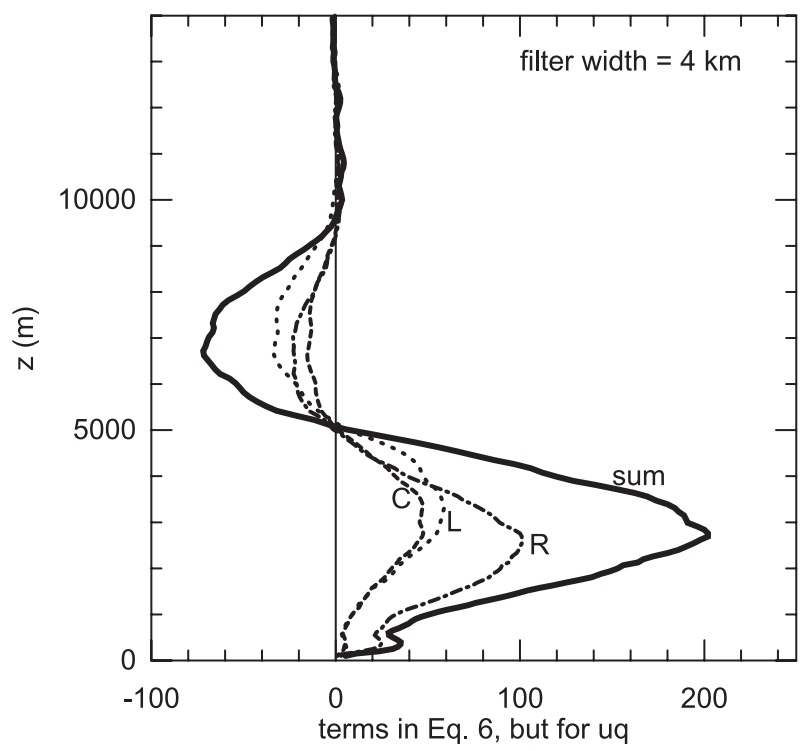

FIG. 11. Vertical distributions of the horizontally averaged $\left\langle\tau_{u q}\right\rangle$ ( $\mathrm{W} \mathrm{m}^{-2}$ ) of the $\mathrm{L}, \mathrm{C}$, and $\mathrm{R}$ terms and their sum, all retrieved from the LES with $\Delta_{f}=4 \mathrm{~km}$.

$$
\tau_{u q}=-K_{h} \frac{\partial \tilde{q}}{\partial x}+2\left(\frac{\Delta_{f}^{2}}{12}\right)\left(\frac{\partial \tilde{u}}{\partial x} \frac{\partial \tilde{q}}{\partial x}+\frac{\partial \tilde{u}}{\partial y} \frac{\partial \tilde{q}}{\partial y}\right)
$$

This model is evaluated, with and without the $\mathrm{L}+\mathrm{C}$ term, in Figs. 12 and 13. The $\mathrm{K}$ term alone correlates very poorly with the LES-retrieved $\tau_{u q}$ and contributes almost nothing to the horizontal mean (Fig. 13) [see also

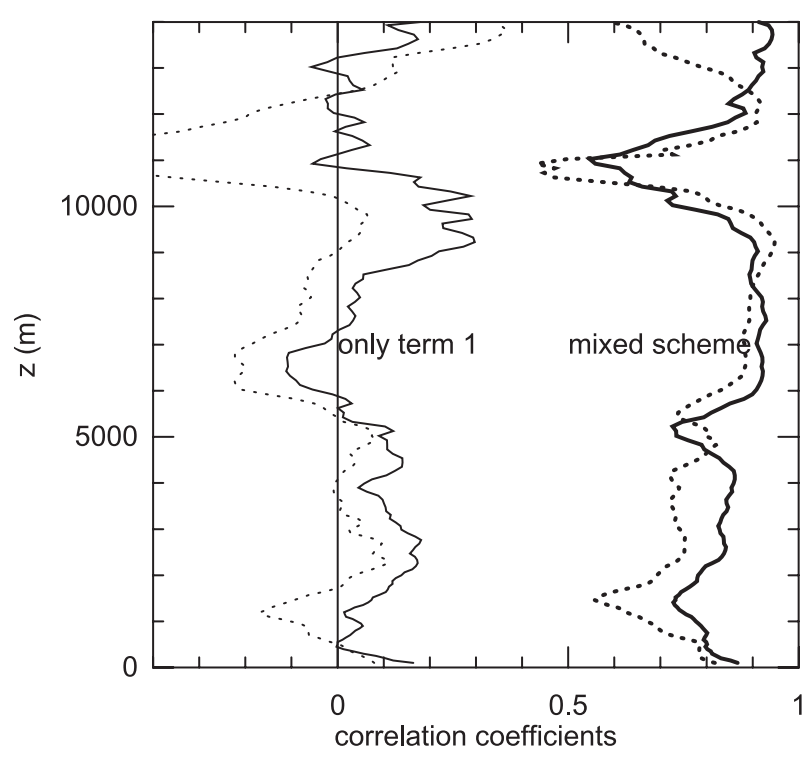

FIG. 12. Correlation coefficients between the modeled and the LES-retrieved $\tau_{u q}$ with $\Delta_{f}=4 \mathrm{~km}$ (solid curves) and $10 \mathrm{~km}$ (dotted curves). 


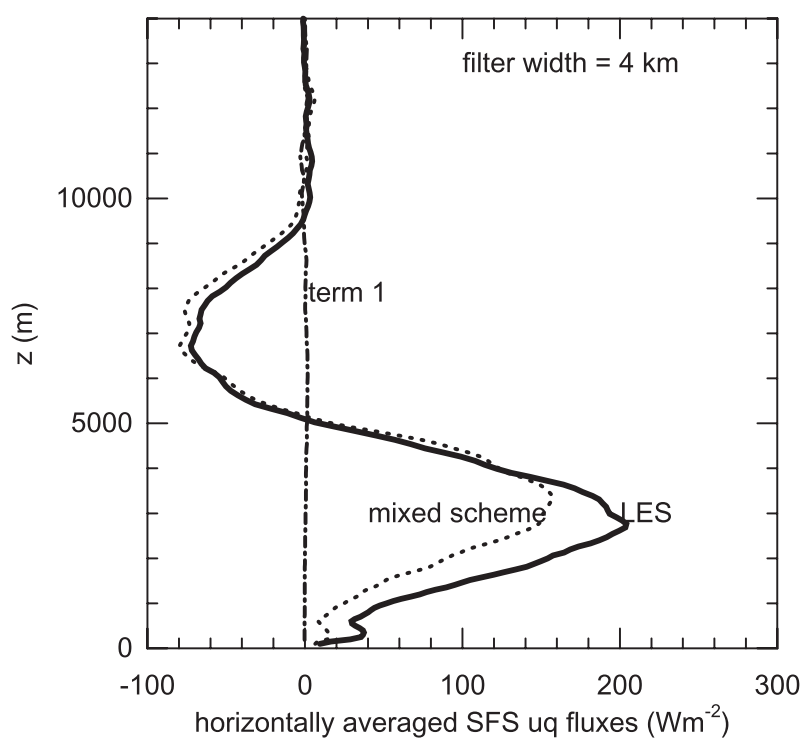

FIG. 13. Vertical distributions of the horizontally averaged $\left\langle\tau_{u q}\right\rangle$ from Eq. (14) in comparison with the LES-retrieved $\left\langle\tau_{u q}\right\rangle$ using $\Delta_{f}=4 \mathrm{~km}$.

the discussion of horizontal scalar flux in Hatlee and Wyngaard (2007)]. Thus, the $K$ model is a poor representation of horizontal SGS fluxes of scalars, even for just the R term.

Including the $\mathrm{L}+\mathrm{C}$ term greatly improves the performance, which yields a spatial distribution that is highly correlated with the LES-retrieved $\tau_{u q}$ (Fig. 12) and also predicts the correct amplitude of the horizontally averaged fluxes (Fig. 13) without any further tuning to the mixed scheme. The horizontal SFS heat flux (not shown) behaves similarly.

\section{c. Vertical SGS flux of zonal wind}

The L, C, and R terms for $\tau_{u w}$ retrieved from the LES are shown in Fig. 14. The R term dominates in the lower and middle cloud layer but otherwise has similar magnitudes relative to the $\mathrm{L}$ and $\mathrm{C}$ terms. The $\mathrm{L}$ and $\mathrm{C}$ terms contribute similarly to the horizontal-mean profile.

To examine the local behavior, we plot the spatial correlation coefficients of the $\mathrm{L}, \mathrm{C}$, and $\mathrm{R}$ terms, individually, with the total SFS flux retrieved from the LES in Fig. 15a; all three components correlate reasonably well with the total $\tau_{u w}$. The correlation coefficient between the $\mathrm{L}$ and $\mathrm{C}$ terms and the ratio of their fluctuation levels are given in Fig. 15b. The correlation between the two terms is not as good as that of the scalar fluxes but is still reasonable, and their fluctuation levels are also similar. This justifies the factor of 2 in the second term of the mixed scheme for $\tau_{u w}$ :

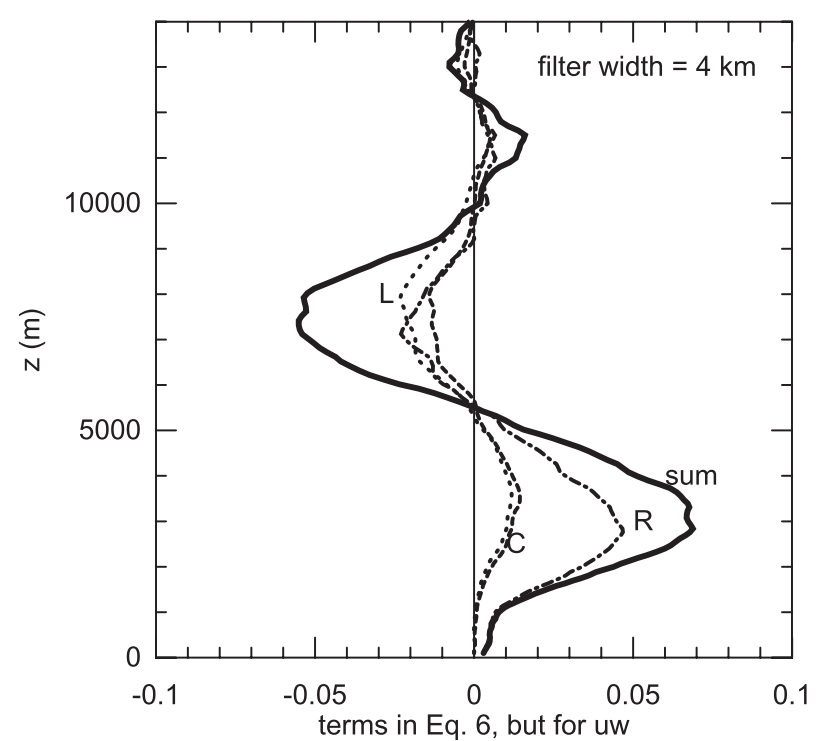

FIG. 14. Vertical distributions of the horizontally averaged $\left\langle\tau_{u w}\right\rangle$ $\left(\mathrm{m}^{2} \mathrm{~s}^{-2}\right)$ of the $\mathrm{L}, \mathrm{C}$, and $\mathrm{R}$ terms in comparison with their sum retrieved from the LES with $\Delta_{f}=4 \mathrm{~km}$.

$$
\tau_{u w}=-K_{m} \frac{\partial \tilde{u}}{\partial z}+2\left(\frac{\Delta_{f}^{2}}{12}\right)\left(\frac{\partial \tilde{u}}{\partial x} \frac{\partial \tilde{w}}{\partial x}+\frac{\partial \tilde{u}}{\partial y} \frac{\partial \tilde{w}}{\partial y}\right) .
$$

The correlation coefficient between the $\mathrm{K}$ term alone and the LES-retrieved $\tau_{u w}$ fluctuates widely in height, with a magnitude close to 0 (Fig. 16). Again the inclusion of the second term in Eq. (15) greatly improves the spatial correlation with the LES-retrieved SFS flux. When compared with the moisture and heat fluxes, the mixed scheme does not perform as well for the momentum flux, particularly for a larger filter width $\Delta_{f}=10 \mathrm{~km}$. This may be due to the effect of gravity waves and pressure dynamics.

The contributions of the two model terms to the horizontally averaged $\tau_{u w}$ are shown in Fig. 17. The K term alone yields much smaller horizontally averaged SGS fluxes and a poor vertical distribution. The magnitude and profile shape from the mixed scheme agree much better with the SFS fluxes retrieved from the benchmark solutions.

\section{Summary and conclusions}

We use a very large domain LES of a tropical convection system as a benchmark to examine the representation of SGS fluxes in CRMs, which have a typical horizontal grid resolution of several kilometers. The domain of this LES is $204.8 \mathrm{~km} \times 204.8 \mathrm{~km} \times 27 \mathrm{~km}$, large enough to cover a mesoscale convection system, while its grid mesh of $100 \mathrm{~m} \times 100 \mathrm{~m}$ in the horizontal plane and $50-100 \mathrm{~m}$ in the vertical direction (for $z<18 \mathrm{~km}$ ) is fine enough to 

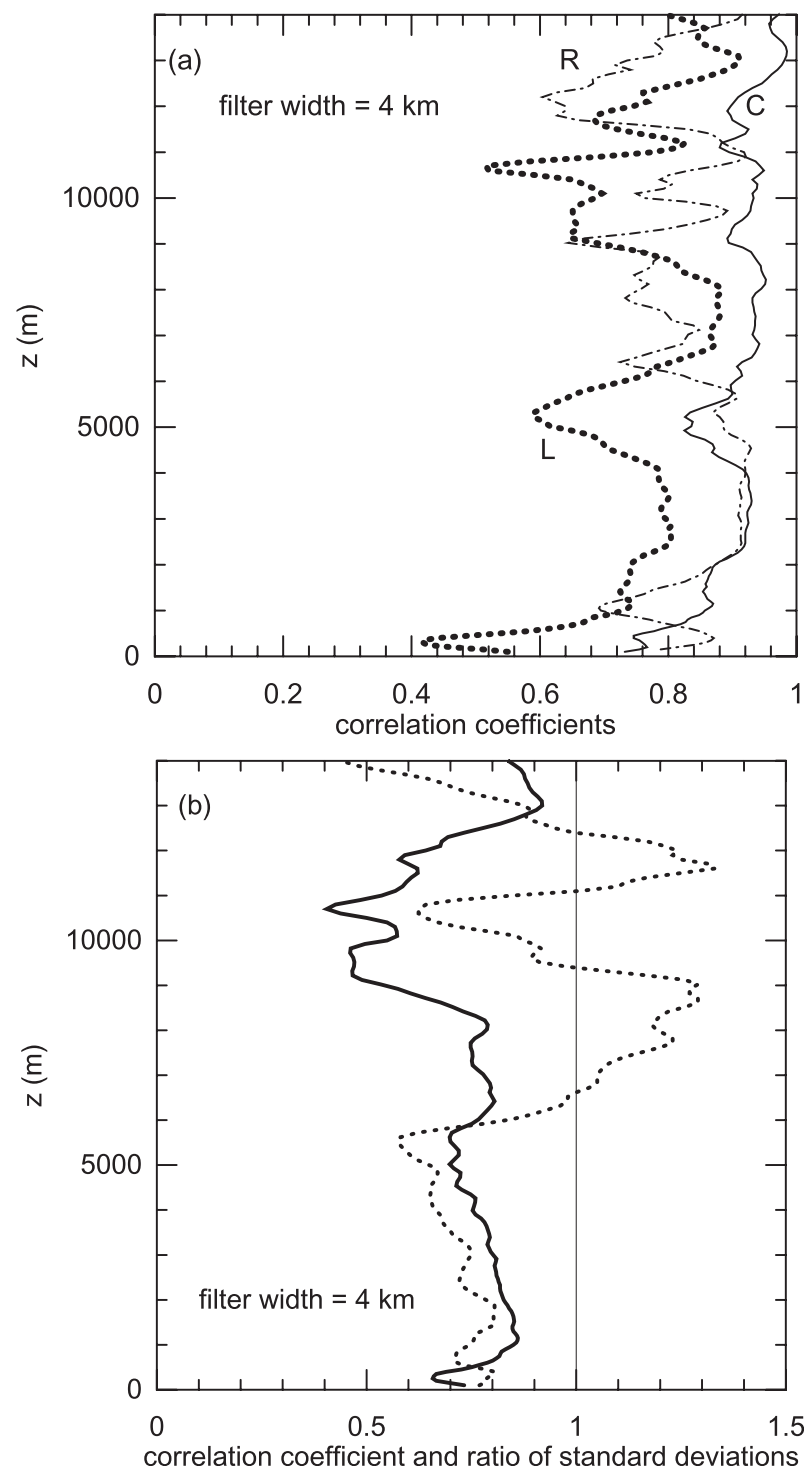

FIG. 15. (a) Correlation coefficients of each individual term with the total SFS flux $\tau_{u w}$, and (b) the correlation coefficient (solid curve) between the $\mathrm{L}$ term and the $\mathrm{C}$ term and the ratio of their standard deviations (dotted curve) retrieved from the LES with $\Delta_{f}=4 \mathrm{~km}$.

resolve energy-containing turbulent motions. The verticalvelocity spectrum (multiplied by wavenumber) peaks over a broad range of scales, from several hundred meters to several kilometers, throughout the deep convection layer except near the surface. This broad spectral peak lies in the range of a typical CRM grid cutoff; there is no spectral gap across the typical CRM grid spacing. The cospectrum between the vertical velocity and moisture fields shows that a large portion of moisture fluxes is carried by motions smaller than a few kilometers, below a typical CRM grid size, throughout the entire deep convection layer.

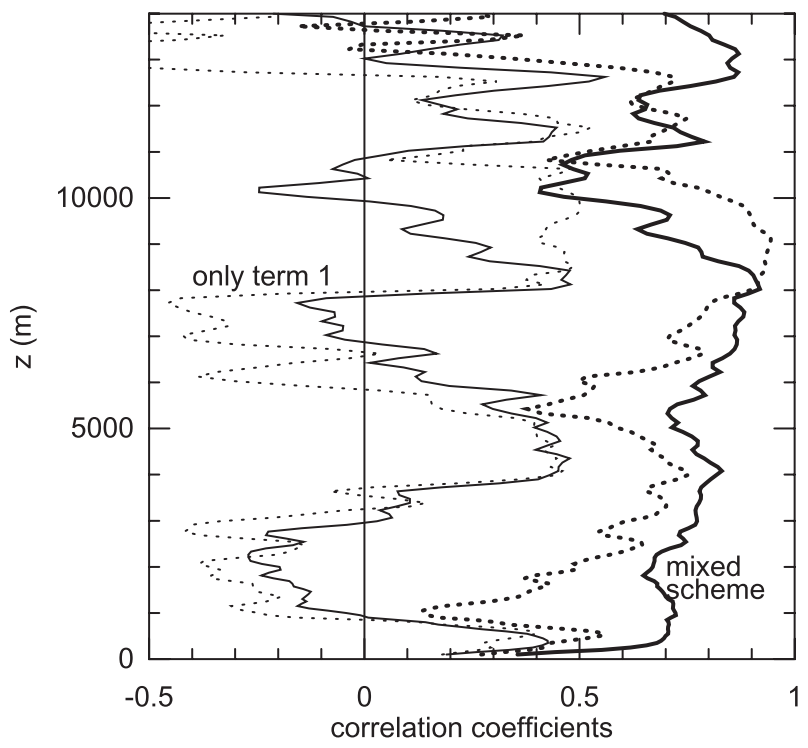

FIG. 16. Correlation coefficients between the modeled and the LES-retrieved $\tau_{u w}$ with $\Delta_{f}=4 \mathrm{~km}$ (solid curves) and $10 \mathrm{~km}$ (dotted curves).

We separate the LES flow field into two components: FS (which is regarded as CRM resolvable scales) and SFS (which is regarded as CRM SGS) using a smooth low-pass filter, and we also retrieve the spatial distributions of the SFS fluxes. With a filter width of $4 \mathrm{~km}$, most of the contribution to the $w$ variance in the benchmark flow is found in the SFS component and about half of the SGS fluxes are carried by the SFS motions. This shows the importance of SGS transport in a typical CRM with a grid spacing of a few kilometers.

To investigate the local relationship of SFS fluxes to FS variables, we further decompose the spatial distributions of SFS fluxes into a Leonard term, a cross term, and a Reynolds term, following Leonard (1974) and Germano (1986). This analysis leads to a mixed SGS flux scheme for CRMs that consists of a commonly used eddy viscosity/diffusivity term and a second term that represents the effect from the largest SFS eddies (i.e., the largest SGS motions, approximately the size of a typical CRM grid resolution). We then perform a priori tests of this scheme against the LES-retrieved SFS fluxes and show that the mixed scheme greatly improves the representation of SGS fluxes of heat, moisture, and momentum for CRMs with a typical grid spacing of a few kilometers.

The a priori tests shown here only provide insight as to how the scheme performs in responding to a given resolvable-scale field (and given SGS TKE distribution). With a priori tests, we cannot speculate as to how it will perform when dynamical adjustments between the SGS fluxes and the CRM resolvable flow are allowed. The 


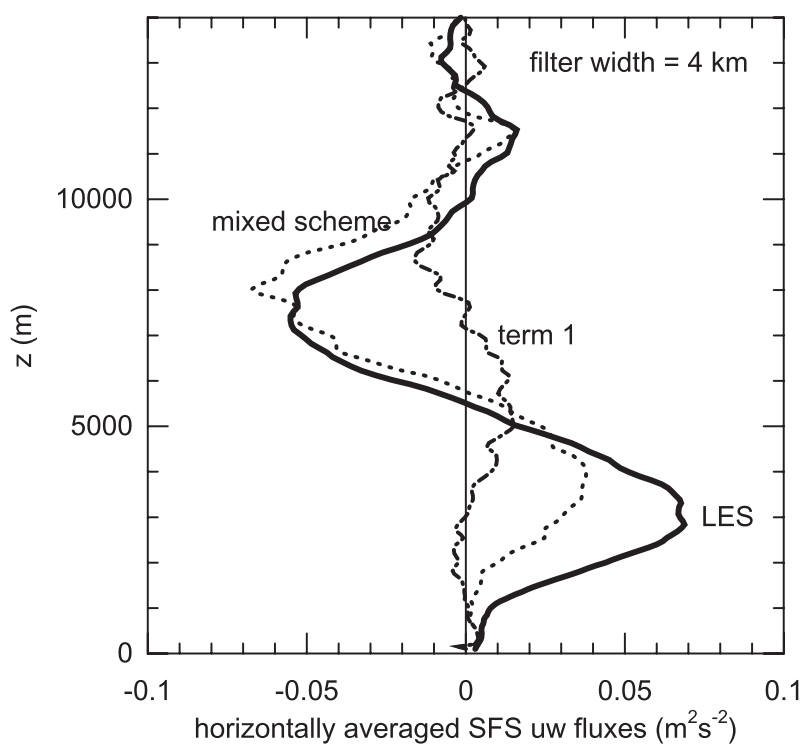

FIG. 17. Vertical distributions of the horizontally averaged $\left\langle\tau_{u w}\right\rangle$ from Eq. (15) in comparison with the LES-retrieved $\left\langle\tau_{u w}\right\rangle$ using $\Delta_{f}=4 \mathrm{~km}$.

ultimate test of the SGS scheme requires performing actual CRM simulations. As a CMMAP project, we plan to implement the mixed SGS scheme into the Colorado State University System for Atmospheric Model, which is a CRM developed by M. Khairoutdinov, to see how the SGS scheme improves CRM simulations of deep convection systems.
Acknowledgments. This material is based on work supported by the National Science Foundation Science and Technology Center for Multi-Scale Modeling of Atmospheric Processes (CMMAP) managed by Colorado State University under cooperative agreement ATM0425247. The LES was performed using the IBM BlueGene/L "New York Blue" supercomputer at the New York Center for Computational Sciences, which is a joint venture of Stony Brook University and Brookhaven National Laboratory.

\section{APPENDIX}

\section{Derivation of Eq. (7)}

For simplicity we demonstrate the derivation using one-dimensional form. Taylor's series expansion of a variable $f(x)$ about a grid point $x_{0}$ yields

$$
\begin{aligned}
f(x)= & f\left(x_{0}\right)+\left(x-x_{0}\right) f^{\prime}\left(x_{0}\right)+\frac{\left(x-x_{0}\right)^{2}}{2} f^{\prime \prime}\left(x_{0}\right) \\
& + \text { higher-order terms. }
\end{aligned}
$$

Here the prime indicates derivatives. The filter-scale $f$ at a point $x_{0}$ is defined as

$$
\tilde{f}\left(x_{0}\right)=\int_{-\infty}^{\infty} G\left(x-x_{0}\right) f(x) d x .
$$

With a $1 \mathrm{D}$ Gaussian filter,

$$
\tilde{f}\left(x_{0}\right)=\sqrt{\frac{6}{\pi}} \frac{1}{\Delta_{f}} \int_{-\infty}^{\infty} \exp \left[-6\left(x-x_{0}\right)^{2} / \Delta_{f}^{2}\right]\left[f\left(x_{0}\right)+\left(x-x_{0}\right) f^{\prime}\left(x_{0}\right)+\frac{\left(x-x_{0}\right)^{2}}{2} f^{\prime \prime}\left(x_{0}\right)+\cdots\right] d x .
$$

Keeping just the first three terms produces

$$
\begin{aligned}
\tilde{f}\left(x_{0}\right) \sim & \sqrt{\frac{6}{\pi}} \frac{1}{\Delta_{f}}\left\{f\left(x_{0}\right) \int_{-\infty}^{\infty} \exp \left[-6\left(x-x_{0}\right)^{2} / \Delta_{f}^{2}\right] d x+f^{\prime}\left(x_{0}\right) \int_{-\infty}^{\infty}\left(x-x_{0}\right) \exp \left[-6\left(x-x_{0}\right)^{2} / \Delta_{f}^{2}\right] d x+f^{\prime \prime}\left(x_{0}\right)\right. \\
& \left.\times \int_{-\infty}^{\infty} \frac{\left(x-x_{0}\right)^{2}}{2} \exp \left[-6\left(x-x_{0}\right)^{2} / \Delta_{f}^{2}\right] d x\right\},
\end{aligned}
$$

which yields

$$
\tilde{f}\left(x_{0}\right) \sim f\left(x_{0}\right)+\frac{\Delta_{f}^{2}}{24} f^{\prime \prime}\left(x_{0}\right) .
$$

To apply Eq. (A5) to Eq. (7), set $f$ to $\tilde{w}, \tilde{c}$, or $\tilde{w} \tilde{c}$.

\section{REFERENCES}

Bardina, J., J. H. Ferziger, and W. C. Reynolds, 1980: Improved subgrid-scale models for large-eddy simulation. American
Institute for Aeronautics and Astronautics Paper 80-1357, $10 \mathrm{pp}$.

Carati, D., G. S. Winckelmans, and H. Jeanmart, 2001: On the modelling of the subgrid-scale and filtered-scale stress tensors in large-eddy simulation. J. Fluid Mech., 441, 119-138.

Chow, F. K., R. L. Street, M. Xue, and J. H. Ferziger, 2005: Explicit filtering and reconstruction turbulence modeling for largeeddy simulation of neutral boundary layer flow. J. Atmos. Sci., 62, 2058-2077.

Clark, R. A., J. H. Ferziger, and W. C. Reynolds, 1979: Evaluation of subgrid-scale models using an accurately simulated turbulent flow. J. Fluid Mech., 91, 1-16. 
Deardorff, J. W., 1980: Stratocumulus-capped mixed layers derived from a three-dimensional model. Bound.-Layer Meteor., 18, 495-527.

Germano, M., 1986: A proposal for a redefinition of the turbulent stresses in the filtered Navier-Stokes equations. Phys. Fluids, 29, 2323-2324.

Gullbrand, J., and F. K. Chow, 2003: The effect of numerical errors and turbulence models in large-eddy simulations of channel flow, with and without explicit filtering. J. Fluid Mech., 495, 323-341.

Hatlee, S. C., and J. C. Wyngaard, 2007: Improved subfilter-scale models from the HATS field data. J. Atmos. Sci., 64, 16941705.

Horst, T. W., J. Kleissl, D. H. Lenschow, C. Meneveau, C.-H. Moeng, M. B. Parlange, P. P. Sullivan, and J. C. Weil, 2004: HATS: Field observations to obtain spatially filtered turbulence fields from crosswind arrays of sonic anemometers in the atmospheric surface layer. J. Atmos. Sci., 61, 1566-1581.

Khairoutdinov, M. F., and D. A. Randall, 2003: Cloud resolving modeling of the ARM summer 1997 IOP: Model formulation, results, uncertainties, and sensitivities. J. Atmos. Sci., 60,607-625.

— , S. K. Krueger, C.-H. Moeng, P. A. Bogenschutz, and D. A. Randall, 2009: Large-eddy simulation of maritime deep tropical convection. J. Adv. Model. Earth Syst., 1, 15, doi:10.3894/ JAMES.2009.1.15.

Klemp, J. B., and R. B. Wilhelmson, 1978: The simulation of threedimensional convective storm dynamics. J. Atmos. Sci., 35, 1070-1096.

Kosovic, B., 1997: Subgrid-scale modelling for the large-eddy simulation of high-Reynolds-number boundary layers. J. Fluid Mech., 336, 151-182.

Leonard, A., 1974: Energy cascade in large-eddy simulations of turbulent fluid flows. Advances in Geophysics, Vol. 18, Academic Press, 237-248.

, 1997: Large-eddy simulation of chaotic convection and beyond. Proc. 35th Aerospace Sciences Meeting, Reno, NV, American Institute for Aeronautics and Astronautics Paper 97-0204.
Liu, S., J. Katz, and C. Meneveau, 1999: Evolution and modelling of subgrid scales during rapid straining of turbulence. J. Fluid Mech., 387, 281-320.

Meneveau, C., and J. Katz, 2000: Scale-invariance and turbulence models for large-eddy simulation. Annu. Rev. Fluid Mech., 32, $1-32$.

Moeng, C.-H., M. A. LeMone, M. F. Khairoutdinov, S. K. Krueger, P. A. Bogenschutz, and D. A. Randall, 2009: The tropical marine boundary layer under a deep convection system: A large-eddy simulation study. J. Adv. Model. Earth Syst., 1, 16, doi:10.3894/JAMES.2009.1.16.

Pielke, R. A., Sr., 2001: Further comments on "The differentiation between grid spacing and resolution and their application to numerical modeling." Bull. Amer. Meteor. Soc., 82, 699-700.

Piomelli, U., P. Moin, and J. H. Ferziger, 1988: Model consistency in large eddy simulation of turbulent channel flows. Phys. Fluids, 31, 1884-1891.

Stolz, S., N. A. Adams, and L. Kleiser, 2001: An approximate deconvolution model for large-eddy simulation with application to incompressible wall-bounded flows. Phys. Fluids, 13, 9971015.

Sullivan, P. P., J. C. McWilliams, and C.-H. Moeng, 1994: A subgridscale model for large-eddy simulation of planetary boundarylayer flows. Bound.-Layer Meteor., 71, 247-276.

— T. T. W. Horst, D. H. Lenschow, C.-H. Moeng, and J. C. Weil, 2003: Structure of subfilter-scale fluxes in the atmospheric surface layer with application to large-eddy simulation modelling. J. Fluid Mech., 482, 101-139.

Tong, C., J. C. Wyngaard, S. Khanna, and J. G. Brasseur, 1998: Resolvable- and subgrid-scale measurement in the atmospheric surface layer: The technique and issues. J. Atmos. Sci., 55, 3114-3126.

Zang, Y., R. L. Street, and J. R. Koseff, 1993: A dynamic mixed subgrid-scale model and its application to turbulent recirculating flows. Phys. Fluids, 5A, 3186-3196.

Zhou, Y., J. G. Brasseur, and A. Juneja, 2001: A resolvable subfilterscale model specific to large-eddy simulation of under-resolved turbulence. Phys. Fluids, 13, 2602-2610. 